\title{
La desnutrición infantil en el medio rural mexicano
}

\author{
Abelardo Avila-C uriel, M.C., M. en M.S., ${ }^{(1)}$ Teresa Shamah-Levy, Lic. en N utr., ${ }^{(2)}$ \\ Carlos Galindo-Gómez, Lic. en N utr., ${ }^{(1)}$ Gerardo Rodríguez-Hernández, Lic. en N utr., ${ }^{(1)}$ \\ Linda M. Barragán-H eredia, Lic. en N utr. ${ }^{(1)}$
}

\section{Avila-Curiel A, Shamah-Levy T, Galindo-Gómez C, Rodríguez-Hernández G, Barragán-Heredia LM. La desnutrición infantil en el medio rural mexicano.} Salud Publica Mex 1998;40:150-160.

\begin{abstract}
Resumen
Objetivo. Conocer la situación nutricional de la población infantil del medio rural mexicano, comparándola con la situación previa y localizando las zonas más afectadas. Ma terial y métodos. La muestra estuvo conformada por 38232 familias, pertenecientes a 855 localidades, seleccionadas probabilísticamente. Se tomaron medidas antropométricas a 31601 menores de cinco años. El análisis se realizó en consideración a la población de referencia peso/ edad, peso/talla y talla/edad. Resultados. La desnutrición global afecta a $42.7 \%$ de los niños de acuerdo con el indicador peso/edad (IC = 1.9\%); hace 22 años las formas de desnutrición moderada y severa eran de $17.4 \%$ y actualmente son de $19.3 \%$. Respecto a talla/edad afecta a $55.9 \%$ (IC $=$ $1.9 \%$ ), y a peso/talla a $18.9 \%$. Conclusiones La EN AL96 muestra que la desnutrición sigue siendo un problema muy grave, que no se ha solucionado y que sigue afectando a las regiones del sur en donde hay mayor prevalencia de grupos indígenas.
\end{abstract}

Palabras clave: trastornos de la nutrición infantil; antropometría; encuestas nutricionales; México

\author{
Avila-Curiel A, Shamah-Levy T, Galindo-Gómez C, \\ Rodríguez-Hernández G, Barragán-Heredia LM. \\ Children malnutrition \\ in rural Mexico. \\ Salud Publica Mex 1998;40:150-160.
}

\begin{abstract}
A bstract
Objective. To evaluate the nutritional status of the infantile population in rural Mexico, compare it with previous data and situate the most affected areas. Material and Methods. The sample consisted of 38232 families, from 855 randomly selected communities. Anthropometry was obtained from 31601 children under five years of age and analysis was performed considering the reference values of weight/age, weight/height and height/age. Results. 0 verall malnutrition affects 42.7 of children according to weight/ age indicator $(\mathrm{Cl}=1.9 \%)$. Moderate and severe malnutrition 22 years ago was $17.4 \%$ and now these affect $19.3 \%$. Further data show that $55.9 \%$ are malnourished according to height/age, and $18.9 \%$ according to weight/height. Conclusions The EN AL 96 shows that malnutrition is still a severe national problem which has not improved in the last 22 years. The areas of highest prevalence are the southern states which have mostly Indian population.
\end{abstract}

Key words: infant nutrition disorders; anthropometry; nutrition surveys; Mexico
$\mathrm{E}$ n los países en vías de desarrollo, la desnutrición infantil se encuentra entre las primeras cinco causas de mortalidad ${ }^{1}$ y se inserta en un contexto de variables sociales, económicas y culturales que además de ser muy desfavorable son, por sí mismas, factores de riesgo que alteran el desarrollo infantil. ${ }^{2}$ En México la desnutrición en menores de cinco años continúa siendo un grave problema de salud pública, a pesar de que durante décadas se han llevado a cabo diversos programas nacionales con el propósito de mejorar la situación. ${ }^{3}$

Recientemente se ha planteado la necesidad de articular las acciones de educación, salud y alimentación, dirigiéndolas integralmente hacia las comunidades

(1) Investigador, Subdirección General de Nutrición, Instituto N acional de la N utrición Salvador Zubirán (IN N SZ), México.

(2) Investigadora, Departamento de Nutrición A plicada y Educación N utricional, IN N SZ, México.

Fecha de recibido: 10 de julio de 1997 • Fecha de aprobado: 15 de enero de 1998

Solicitud de sobretiros: Dr. A belardo Avila Curiel.Tlalpan s/n, Unidad de Investigación, edificio C 201, colonia Tlalpan, 14080 México, D. F. Correo electrónico: aavila@mex1.uninet.net.mx 
indígenas, las zonas rurales y urbanas marginadas y, particularmente, a los miembros más vulnerables de las familias pobres: los menores de cinco años y las mujeres embarazadas y en periodo de lactancia. ${ }^{4} \mathrm{Un}$ requisito indispensable para la planeación, el funcionamiento y la evaluación de la política de bienestar social y de los programas de alimentación y nutrición es el diagnóstico objetivo de las condiciones de alimentación y nutrición de la población, especialmente en el medio rural.

A partir de 1958 el Instituto Nacional de la Nutrición Salvador Zubirán (INNSZ) inició el estudio sistemático de las características, la distribución y la magnitud de la desnutrición en México; ${ }^{5}$ en 1974 aplicó la primera Encuesta Nacional de Alimentación en el Medio Rural Mexicano (ENAL-74) 6 a 10772 familias de 90 comunidades rurales con menos de 2500 habitantes, representativas de las 90 regiones geoeconómicas en las que se dividió el país de acuerdo con la regionalización propuesta por Bassols. ${ }^{7}$ Se obtuvo información antropométrica de talla y perímetro mesobraquial de 7108 niños de entre 1 y 5 años. La información de esta encuesta sólo se procesó en una mínima parte y permaneció inédita hasta 1990, sin que haya tenido un efecto real en la planeación de estrategias ni en la toma de decisiones de los programas y acciones relacionados con la alimentación y la nutrición popular.

En 1979 se realizó el levantamiento de la segunda Encuesta Nacional de Alimentación en el Medio Rural Mexicano (ENAL-79) bajo la coordinación del INNSZ y con la participación operativa del Instituto Nacional Indigenista (INI) y los Servicios Coordinados de Salud en los Estados de la Secretaría de Salubridad y Asistencia (SSA) ${ }^{8,9}$ Con esta encuesta se estudiaron 21248 familias de 219 comunidades rurales, esto es, las 90 comunidades encuestadas en 1974 más 129 seleccionadas aleatoriamente en proporción a la población de las 90 regiones. Se obtuvo información antropométrica de talla y perímetro mesobraquial en 11500 niños de entre 1 y 5 años de edad.

En el periodo 1982-1988 se produjo un vacío de información casi completo en materia de vigilancia epidemiológica de la nutrición. En 1988 la Dirección General de Epidemiología de la Secretaría de Salud (SSA) realizó el levantamiento de la Encuesta Nacional de Nutrición (ENN-88). ${ }^{10}$ Debido al diseño muestral de la encuesta sólo fue posible desagregar la información antropométrica de alrededor de 7400 niños en cuatro grandes regiones geográficas del país sin diferenciación urbano-rural. Dado que dichas regiones presentan notables contrastes socioeconómicos en su interior, esta encuesta, si bien permitió obtener una estimación estadísticamente confiable de la prevalencia de desnutrición en la población menor de cinco años en el país, impidió, por otra parte, apreciar su magnitud en la población en situación de mayor riesgo. Los indicadores antropométricos informados por esta encuesta incluyeron peso para la edad, talla para la edad y peso para la talla en relación con la población de referencia de la Organización Mundial de la Salud/Centro Nacional de Estadísticas en Salud (OMS/NCHS).

En 1989 se llevó a cabo la tercera Encuesta Nacional de Alimentación en el Medio Rural Mexicano (ENAL-89), ${ }^{11}$ la cual se desarrolló bajo los auspicios de la Comisión Nacional de Alimentación. La coordinación técnica estuvo a cargo del INNSZ, y la operación en campo fue ejecutada por el INI, por los servicios estatales de salud de la SSA, mediante el Programa de Nutrición y Salud de la Dirección General de Salud Materno-Infantil, y por el Instituto Mexicano del Seguro Social-Solidaridad (IMSS-Solidaridad); se contó, además, con el apoyo del Sistema Nacional para el Desarrollo Integral de la Familia (DIF). Esta encuesta se concibió como una réplica de la ENAL-79, y su aplicación en las mismas comunidades proporcionó una visión dinámica de la evolución de las condiciones de nutrición de la población en el medio rural. ${ }^{12}$ Con la ENAL-89 se obtuvo información de 20759 familias de las mismas localidades estudiadas por la ENAL-79. Se registró el peso, la talla y el perímetro de brazo de 15400 niños menores de cinco años. Los indicadores antropométricos y la población de referencia fueron los que se utilizaron en la ENN-88.

Además de estas encuestas están otros estudios recientes que permiten estimar la prevalencia y la distribución de los problemas de desnutrición en México. En 1993 y 1994 el DIF realizó el Primer y el Segundo Censo Nacional de Talla en Escolares, ${ }^{13,14}$ respectivamente, entre todos los niños de primer año de primaria en el país. Estos censos brindan una buena estimación del estado de nutrición de los niños referido a los años anteriores al levantamiento; la prevalencia de tallas bajas se correlaciona consistentemente con la prevalencia de desnutrición crónica, pero es poco lo que indica acerca del estado actual de nutrición de los niños. En 1995 el INNSZ realizó la Encuesta Urbana de Alimentación en la Zona Metropolitana de la Ciudad de México, ${ }^{15}$ mediante la cual quedó manifiesta la predominancia de problemas nutricionales por exceso y desequilibrio en la alimentación de la población urbana, si bien se detectó un importante número de niños prescolares desnutridos entre la población urbana marginada.

Desde 1989 no había un estudio que permitiera conocer la distribución de la desnutrición en la población prescolar del medio rural mexicano. Este vacío de 
información constituye, sin duda, un serio obstáculo para la planeación más eficaz en los programas de nutrición y desarrollo social orientados a fomentar el bienestar social de la población en condiciones de marginación. En consideración a que las encuestas previas aportaron la información más consistente y comparable que se tenía para apreciar los niveles de desnutrición, su distribución geográfica y su evolución en los años recientes, se consideró pertinente realizar la Cuarta Encuesta Nacional de Alimentación en el Medio Rural Mexicano (ENAL-96), cuyo objetivo primordial fue conocer la situación actual alimentaria y nutricional en el medio rural mexicano e identificar factores de riesgo asociados con desnutrición infantil en las comunidades y familias estudiadas.

En el presente trabajo se expone exclusivamente el análisis primario de los resultados de la ENAL 96 mediante la medición antropométrica de peso, talla y edad en la población menor de cinco años. Los resultados se presentan por entidad federativa, y de acuerdo con la condición indígena de las localidades, así como con la correspondencia entre algunas variables socioeconómicas seleccionadas y la prevalencia de desnutrición estatal. Se presenta también una comparación de la prevalencia de desnutrición observada a partir de las cuatro encuestas de la serie. El análisis de los factores de riesgo asociados, así como de las diferencias regionales por sexo y grupos de edad serán objeto de futuros trabajos.

\section{Material y métodos}

La ENAL-96 recabó información referente a las características socioeconómicas de las familias, los indicadores antropométricos de los prescolares, la lactancia, la ablactación y la morbilidad, los indicadores de fecundidad y la mortalidad infantil, y el consumo familiar de alimentos, mediante una muestra probabilística. El universo que abarcó la encuesta estuvo conformado por todas las familias residentes en las localidades rurales del país con población de 500 a 2500 habitantes y cuya población económicamente activa estuviera dedicada sobre todo a la agricultura, de acuerdo con la información del X Censo Nacional de Población y Vivienda, 1990.

Las localidades se asignaron a estratos (regiones) al interior de cada entidad federativa. Estos estratos se construyeron respetando siempre los contornos de la división municipal, así como, en la medida de lo posible, las regionalizaciones político-administrativas locales. La unidad mínima de desagregación fue el municipio y siempre que las circunstancias lo permitieron, se trató de identificar un estrato con un municipio, como sucedió en los estados de Tabasco, Baja California, Baja California Sur, Campeche y Sinaloa. En el caso de Oaxaca, que contiene la cuarta parte de los municipios del país, se determinó identificar las regiones con los treinta distritos administrativos del estado. Para la conglomeración de los restantes municipios se utilizaron criterios socioeconómicos, geográficos, etnográficos, fisiográficos, de lugar central, contigüedad territorial y vías de comunicación. Se definieron 372 estratos para la aplicación de la encuesta.

La unidad primaria de muestreo estuvo conformada por las localidades de cada municipio o conjunto de municipios que conformaron lo estratos. En cada estrato se seleccionó aleatoriamente un mínimo de dos localidades y, en algunos casos, dependiendo de la capacidad institucional para el trabajo de campo, fueron tres las localidades seleccionadas, siempre al azar. La ENAL-96 se aplicó en 855 localidades.

En cada localidad se elaboró un marco muestral con el listado de todas las familias, a partir del cual se selecciónó, mediante muestreo aleatorio simple, a 50 familias. La unidad de aplicación de la encuesta fue la familia, entendida ésta como el conjunto de tres o más personas que, unidas o no por parentesco, viven bajo el mismo techo, y comparten el gasto y la alimentación doméstica. El número de familias por encuesta se estableció en función de la capacidad operativa de los equipos de levantamiento de la misma.

Se excluyó de la muestra a las familias imposibles de localizar en las comunidades durante la permanencia del equipo de encuestadores, así como a las que se negaron a participar y a aquellas en las que no se encontró una persona capaz de proporcionar la información requerida. Esos casos no se sustituyeron por otras familias y se notificaron las razones de no levantamiento en el informe comunitario.

Se aplicó un total de 38232 encuestas en las familias y se tomaron medidas antropométricas de 31601 menores de cinco años. Para el análisis del presente trabajo se excluyeron los valores antropométricos considerados aberrantes: mayores y menores de +5 y -5 desviaciones estándar respectivamente, de acuerdo con la población de referencia de los indicadores peso para la edad, peso para la talla y talla para la edad.

Para la aplicación de la encuesta, en la mayoría de los estados se mantuvo la comparabilidad estacional de la serie de Encuestas Nacionales de Alimentación, es decir, que el trabajo de campo se desarrolló entre los meses de agosto y diciembre.

El encuestador se presentó ante cada familia, explicó claramente el objetivo de la encuesta y comunicó a cada entrevistado que la información proporcionada era confidencial individual y familiarmente. El cues- 
tionario se aplicó al ama de casa, al jefe de familia o a la persona responsable de la familia. Antes del levantamiento de la ENAL-96 se realizó una prueba piloto en comunidades rurales del estado de Morelos.

El personal estandarizado del INNSZ se responsabilizó, a su vez, de la capacitación y la estandarización del personal de campo de toda la República. Personal del DIF en los estados, del IMSS-Solidaridad, del INI y del propio INNSZ llevó a cabo las entrevistas; en algunos estados participó la SSA y se contó con el apoyo logístico de la Secretaría de Desarrollo Social. Para la toma de medidas antropométricas se procedió a la estandarización del personal de campo mediante la metodología propuesta por Habitch. ${ }^{16}$ El equipo que se utlizó para realizar las mediciones antropométricas de los prescolares constó de infantómetros Dynatop con precisión de $0.5 \mathrm{~cm}$, estadímetros de escuadra y flexómetro Dynatop con precisión de $1 \mathrm{~mm}$, y básculas tipo Salter de $20 \mathrm{~kg}$ de capacidad y precisión de $100 \mathrm{~g}$.

La información se capturó por duplicado y mediante un programa de validación de rangos de las variables. Un equipo de nutriólogos y los investigadores del INNSZ responsables de la encuesta se encargaron de codificar y procesar la información; esta última se analizó mediante frecuencias, medias, varianza, intervalos de confianza al $95 \%$ y los factores de expansión de las viviendas (número de viviendas en la localidad/ número de encuestas realizadas en la localidad) y de las localidades (número de localidades del estrato/ número de localidades encuestadas en el estrato).

La estimación del estado de nutrición de los prescolares se consignó de acuerdo con cuatro indicadores clásicos: a) peso para la edad; b) talla para la edad; c) peso para la talla, y d) clasificación de Gómez.

Las categorías de los tres primeros corresponden a la recomendación de la Organización Mundial de la Salud (OMS), la cual considera como normales a los niños con valores antropométricos iguales o mayores a -1 puntuación $Z$; con desnutrición leve cuando el rango se ubica entre menor de $-1 Z$ y mayor de $-2 Z$; moderada entre menor o igual a -2 y mayor que $-3 Z$, y severa cuando el valor antropométrico se ubica por debajo de $-3 \mathrm{Z}$ de la población de referencia OMS/ NCHS. ${ }^{17}$

La clasificación de Gómez considera normales a los niños cuyo peso es mayor o igual a $90 \%$ del valor de la mediana del peso para la edad y el sexo en la población de referencia; el grado I de desnutrición corresponde a déficits superiores a $10 \%$ e inferiores a $25 \%$; el grado II se ubica entre 25 y $39.9 \%$, y el grado III es superior a $40 \%$.

La comparación de la prevalencia de desnutrición en niños de 1-5 años, según el estimador peso para la edad que se obtuvo en la serie ENAL, requirió del reprocesamiento de las bases de datos correspondientes a las dos primeras encuestas de la serie. En la ENAL-74 y en la ENAL-79 se incluyó la evaluación del estado de nutrición la cual se llevó a cabo mediante la medición del perímetro mesobraquial y la tallade exclusivamente, niños de 1-5 años. La ENAL-89 registró peso, talla y perímetro mesobraquial en todos los niños menores de cinco años. Mediante un modelo de regresión por grupos de edad y sexo, de las mediciones del perímetro braquial, el peso y la puntuación $Z$ del peso para la edad, obtenidos en la ENAL-89, se pudieron estimar los equivalentes del perímetro mesobraquial en relación con el estado de nutrición obtenido por la medición directa del peso para la edad en los niños de 1-5 años. El perímetro mesobraquial no mostró capacidad para discriminar en forma confiable a los desnutridos moderados de los severos, por lo que ambas categorías se agrupan. A partir de estos valores se volvió a procesar la información de las dos primeras encuestas para estimar la prevalencia de desnutrición de acuerdo con el peso para la edad y poder comparar las tres encuestas. A estos resultados anteriormente notificados se agregan los de la ENAL-96.

El procesamiento estadístico se realizó por medio del programa SPSS/Windows $95,{ }^{18}$ y la información antropométrica se procesó mediante el programa Sistema de Cálculo Antropométrico del Instituto Nacional de la Nutrición (SCAIN).

\section{Resultados}

En el cuadro I se muestra la distribución porcentual por entidad federativa de la población menor de cinco años de acuerdo con el indicador peso para la edad. La prevalencia de desnutrición fue de $42.7 \%$ (intervalo de confianza $-\mathrm{IC}-=1.9 \%$ ). La forma leve de desnutrición afectó a $25.9 \%$ de los niños $(\mathrm{IC}=1.16 \%)$, la moderada a $12.7 \%(\mathrm{IC}=0.9 \%)$ y la forma severa a $4.2 \%$ (IC $=1.5 \%$ ). Llama la atención el contraste de los niveles de desnutrición entre las diversas entidades: los estados de Guerrero, Yucatán, Puebla, Oaxaca y Chiapas presentan una prevalencia de desnutrición moderada y severa superior a $20 \%$, mientras que en Tamaulipas, Sinaloa, Jalisco, Durango, Coahuila, Baja California y Sonora, ésta es inferior a $8 \%$.

En el cuadro II se muestra la distribución porcentual del estado de nutrición por entidad federativa de acuerdo con el indicador talla para la edad. La prevalencia de desnutrición fue de $55.9 \%$ ( $\mathrm{IC}=1.9 \%$ ). Las formas moderadas y severas afectaron a $33.8 \%$ de los niños (IC 2.2\%). Al igual que con el estimador peso para la edad, se observa un notable contraste entre las 


\section{Cuadro I \\ Distribución del estado de nUtRición en la población menor de Cinco años en localidades DE $\mathbf{5 0 0}$ A 2500 HABITANTES, POR ENTIDAD FEDERATIVA, DE ACUERDO CON EL ESTIMADOR ANTROPOMÉTRICO Peso para la edAd. México, 1996}

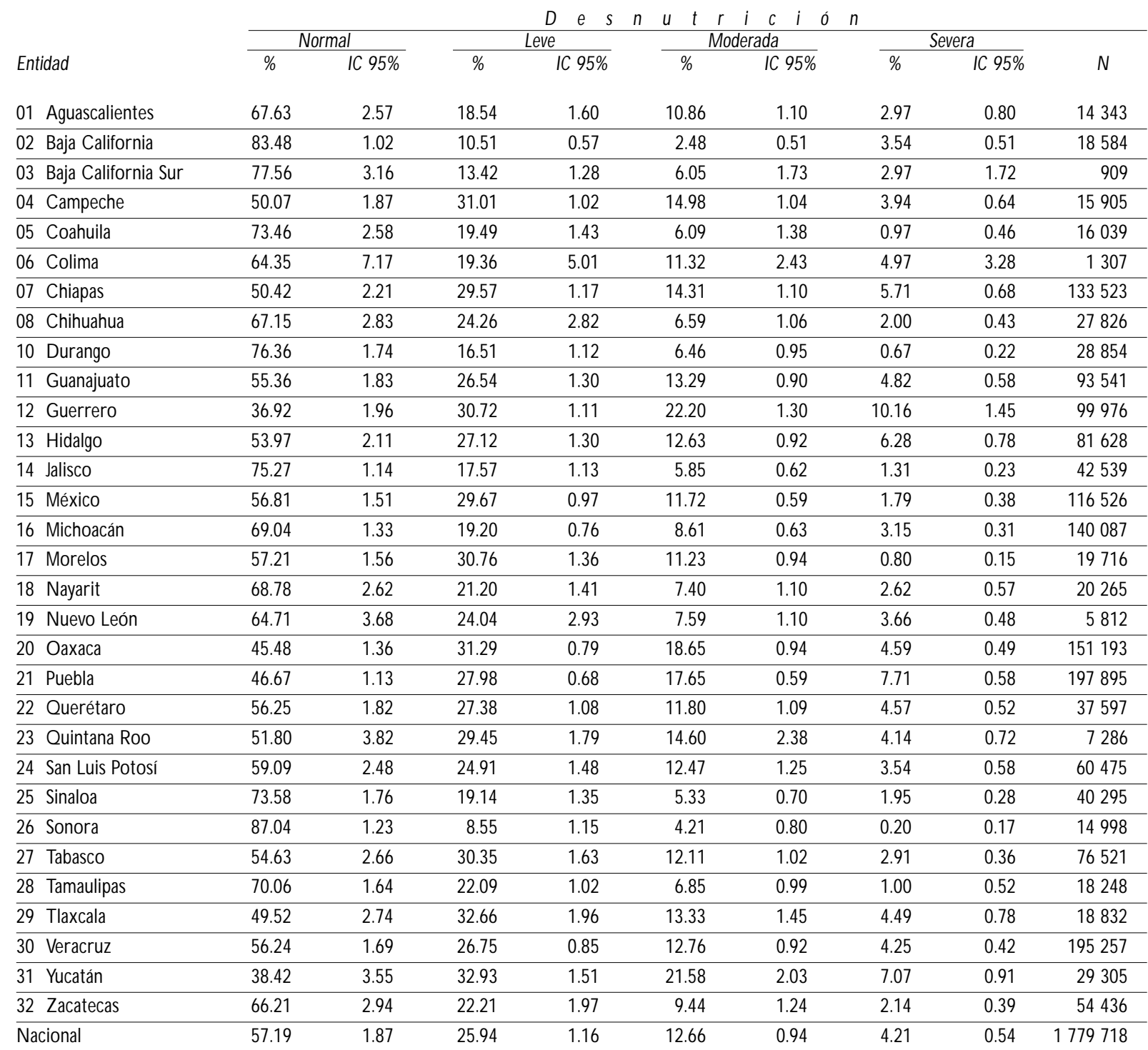

Fuente: Instituto N acional de la N utrición Salvador Zubirán, EN AL-1996

entidades federativas: Yucatán, Chiapas, Guerrero, Campeche, Oaxaca, Quintana Roo y Puebla presentan una prevalencia de desnutrición moderada y severa superior a $40 \%$, mientras que en Tamaulipas, Chihuahua, Coahuila, Jalisco, Sinaloa, Sonora y Baja California ésta es inferior a $20 \%$.

En el cuadro III se muestra el estado de nutrición de los niños menores de cinco años según el indicador peso para la talla. La prevalencia de desnutrición en el país es de $18.9 \%: 11.8 \%$ en grado leve, $4.8 \%$ moderado y $2.3 \%$ en grado severo. Los estados que presentan mayores niveles de desnutrición, según este indicador, son Nuevo León, Nayarit, Guerrero, Guanajuato y Yucatán, con prevalencias de alrededor de $25 \%$, mientras que la prevalencia inferior a $14 \%$ se observa en Campeche, Baja California, Morelos y Michoacán.

De acuerdo con la clasificación de Gómez, la prevalencia de desnutrición en el país es de 46.4\%: 36.1\% 


\section{Cuadro II \\ Distribución del estado de nUtrición en la población MENOR de CinCo años en localidades DE 500 A 2500 HABITANTES, POR ENTIDAD FEDERATIVA, DE ACUERDO CON EL ESTIMADOR ANTROPOMÉTRICO talla PARA la EdAD. MÉXico, 1996}

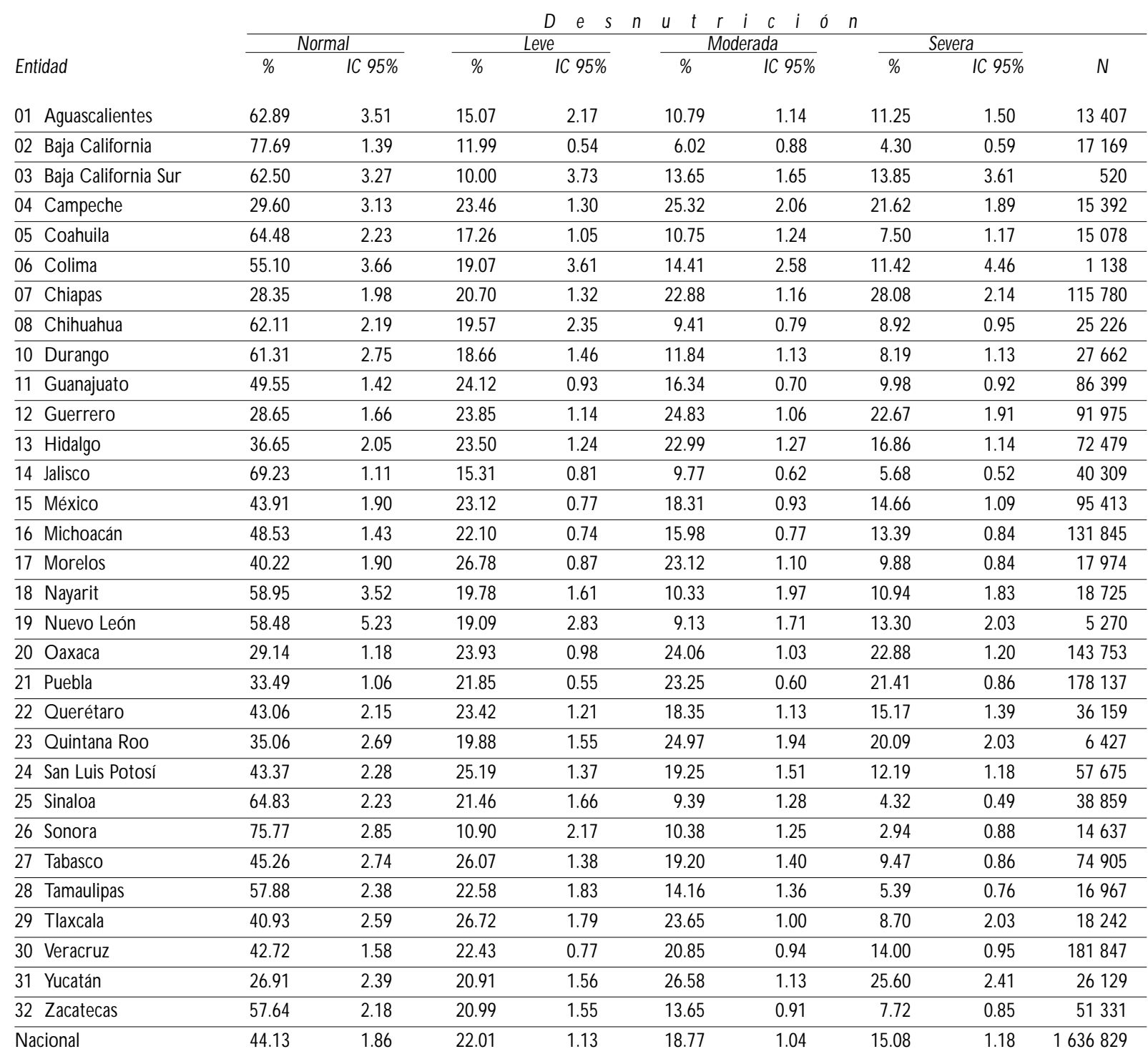

Fuente: Instituto N acional de la N utrición Salvador Zubirán, EN AL-96

en primer grado, $9 \%$ en segundo, y $1.3 \%$ en grado tres. Los niveles más altos de desnutrición se observaron en Guerrero, Yucatán, Campeche, Oaxaca, Puebla y Chiapas, en tanto que la menor prevalencia se registró en Sonora, Baja California, Baja California Sur, Durango y Jalisco.

Al observar la prevalencia por entidad federativa destaca el hecho de que los estados con mayor población indígena son los que presentan mayores niveles de desnutrición. Al analizar la prevalencia de desnutrición de acuerdo con la condición indígena de las comunidades estudiadas se hace evidente la gran diferencia, dependiente de tal condición. En el cuadro IV se presenta la prevalencia de desnutrición de acuerdo con los cuatro indicadores, diferenciando a la población en tres estratos: si en la comunidad de residencia la mayoría de la población habla alguna lengua indígena (comunidad indígena); si hay una minoría que 


\section{Cuadro III \\ LoCALIDADES DE $\mathbf{5 0 0}$ a $\mathbf{2} \mathbf{5 0 0}$ HABITANTES, POR ENTIDAD FEDERATIVA, de acuerdo con el estimador antropométrico peso para la talla. México, 1996}

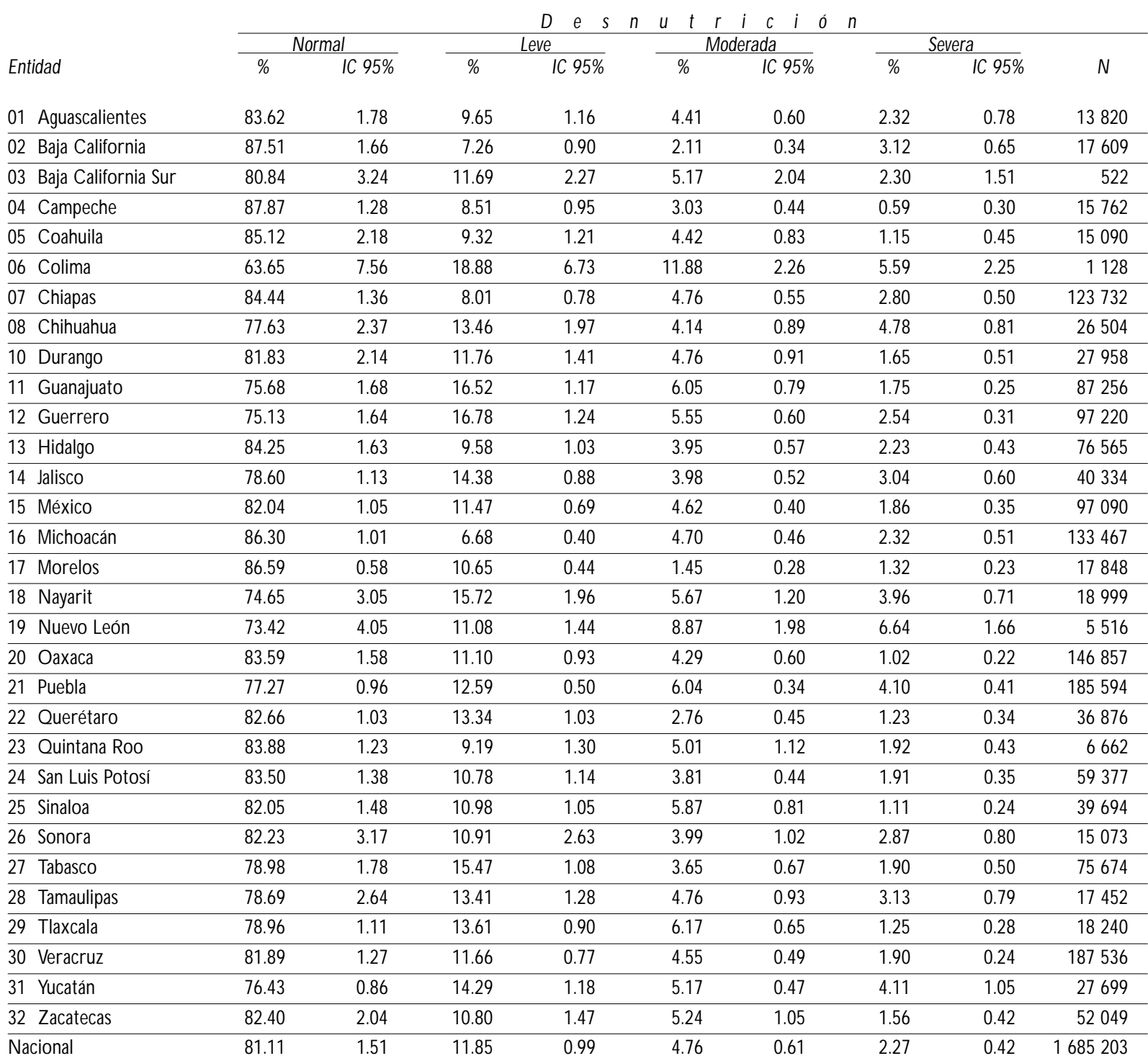

Fuente: Instituto N acional de la N utrición Salvador Zubirán, EN AL-96

la hable (comunidad con presencia indígena), y si no hay hablantes indígenas (comunidad no indígena). De acuerdo con el indicador peso para la edad, en las comunidades no indígenas la prevalencia de desnutrición en la población menor de cinco años es de $38.5 \%$; en las comunidades con presencia indígena es de $45.2 \%$, y se eleva hasta $58.3 \%$ en las comunidades indígenas. La prevalencia de desnutrición moderada y severa es de $14.1,17.3$ y $28.2 \%$, respectivamente. De acuerdo con el indicador talla para la edad, la desnu- trición afecta a 50.9\% de los niños de las comunidades no indígenas, a $59.5 \%$ en las comunidades con presencia indígena, y a 73.6\% de los niños de las comunidades indígenas.

El cuadro $\mathrm{V}$ presenta la comparación de la prevalencia de desnutrición en niños de 1-5 años según el estimador peso para la edad obtenida por la serie ENAL. En el plano nacional resulta sorprendente la similitud de la prevalencia estimada en las cuatro encuestas. De 1974 a 1996 la prevalencia de desnutrición 


\section{Cuadro IV \\ Estado de NUtRICIÓN DE LA POBLACIÓN MENOR DE CINCO AÑOS SEGÚN CONDICIÓN INDÍGENA}

\begin{tabular}{|c|c|c|c|c|c|}
\hline & $\begin{array}{c}\text { Normal } \\
\%\end{array}$ & $\begin{array}{l}\text { Leve } \\
\%\end{array}$ & $\begin{array}{c}\text { Moderada } \\
\%\end{array}$ & $\begin{array}{c}\text { Severa } \\
\%\end{array}$ & $\mathrm{~N}$ \\
\hline $\begin{array}{l}\text { Peso/edad } \\
\text { Indígenas } \\
\text { Presencia indígena } \\
\mathrm{N} \text { o indígenas } \\
\text { Global }\end{array}$ & $\begin{array}{l}41.73 \\
54.78 \\
61.49 \\
55.87\end{array}$ & $\begin{array}{l}30.03 \\
27.91 \\
24.47 \\
26.33\end{array}$ & $\begin{array}{l}20.05 \\
12.98 \\
10.88 \\
13.31\end{array}$ & $\begin{array}{l}8.19 \\
4.33 \\
3.16 \\
4.49\end{array}$ & $\begin{array}{r}6614 \\
5223 \\
17680 \\
29517\end{array}$ \\
\hline $\begin{array}{l}\text { Peso/talla } \\
\text { Indígenas } \\
\text { Presencia indígena } \\
\text { No indígenas } \\
\text { Global }\end{array}$ & $\begin{array}{l}81.20 \\
81.75 \\
81.02 \\
81.19\end{array}$ & $\begin{array}{l}11.56 \\
11.00 \\
12.21 \\
11.85\end{array}$ & $\begin{array}{l}4.98 \\
4.45 \\
4.59 \\
4.65\end{array}$ & $\begin{array}{l}2.26 \\
2.80 \\
2.18 \\
2.31\end{array}$ & $\begin{array}{r}6411 \\
4856 \\
16789 \\
28056\end{array}$ \\
\hline $\begin{array}{l}\text { Talla/edad } \\
\text { Indígenas } \\
\text { Presencia indígena } \\
\text { No indígenas } \\
\text { Global }\end{array}$ & $\begin{array}{l}26.39 \\
40.52 \\
49.12 \\
42.58\end{array}$ & $\begin{array}{l}21.85 \\
23.36 \\
22.39 \\
22.44\end{array}$ & $\begin{array}{l}25.55 \\
20.09 \\
16.83 \\
19.33\end{array}$ & $\begin{array}{l}26.21 \\
16.02 \\
11.66 \\
15.65\end{array}$ & $\begin{array}{r}6059 \\
4738 \\
16483 \\
27280\end{array}$ \\
\hline $\begin{array}{l}\text { Gómez } \\
\text { Indígenas } \\
\text { Presencia indígena } \\
\text { No indígenas }\end{array}$ & $\begin{array}{l}38.23 \\
51.02 \\
57.92\end{array}$ & $\begin{array}{l}42.98 \\
38.93 \\
33.76\end{array}$ & $\begin{array}{r}16.46 \\
8.75 \\
7.36\end{array}$ & $\begin{array}{l}2.34 \\
1.30 \\
0.96\end{array}$ & $\begin{array}{r}6636 \\
5233 \\
17703\end{array}$ \\
\hline N acional & 52.28 & 36.74 & 9.65 & 1.33 & 29572 \\
\hline
\end{tabular}

Fuente: Instituto N acional de la N utrición Salvador Zubirán, EN AL-1996

se ubica alrededor de 50\%; la leve, alrededor de 30\%, y la moderada y severa, en torno a $20 \%$. Como se indicó con anterioridad, la estrategia de muestreo de las primeras tres versiones de la serie ENAL difiere de esta cuarta versión en cuanto a regionalización. En las tres primeras las comunidades seleccionadas se asignaron a 19 zonas a las cuales podrían pertenecer municipios de varios estados, mientras que en la ENAL-96 se asignaron a cada estado.

Como ejercicio exploratorio, se asignaron las comunidades seleccionadas aleatoriamente por la ENAL-96 a las 19 zonas de la serie y se estimó su prevalencia de desnutrición de acuerdo con el indicador peso para la edad, de tal manera que se pudiera hacer una primera comparación por zona, sin dejar de advertir las limitaciones de este procedimiento. De acuerdo con este ejercicio, las nueve zonas más septentrionales del país (1 a 9) presentaron en 1996 una prevalencia de desnutrición entre 10 y 36\%, y la mayoría mostró una evolución consistentemente favorable desde niveles de prevalencia de desnutrición bajos en 1974. Aunque en 1996 a excepción de la zona 8, todas tuvieron una prevalencia de desnutridos moderados y severos in- ferior a 10\% -la más baja del país-, en siete de estas zonas dicha prevalencia fue superior a la de 1989.

Las zonas 10 y 12 en el centro del país, así como las 15 y 18 en el Golfo de México, presentan una discreta evolución favorable: prevalencia de desnutrición global menor a $50 \%$, y moderada y severa de menos del 20\%. Las zonas 11 (Huasteca y Sierra Norte de Puebla), 16 (Mixteca) y 17 (Tehuantepec y sur de Chiapas) presentaron en 1996 niveles elevados de desnutrición (entre 55 y 62\%), pero notablemente inferiores a los registrados en la ENAL-89 (alrededor de 70\%). Lo mismo ocurre con la zona 19 (Península de Yucatán), aunque en menor grado.

Finalmente, en las zonas 13 (Guerrero y Michoacán) y la 14 (Tlaxcala y sur de Puebla) se observa un deterioro importante entre 1989 y 1996. En estas zonas la prevalencia de desnutrición es superior a $55 \%$ y, en ambos casos, las formas moderadas y severas se incrementaron de 16 a $25 \%$.

\section{Discusión}

En términos epidemiológicos se recomienda utilizar el indicador peso para la edad como el estimador más fehaciente de la prevalencia actual de desnutrición en los niños menores de cinco años; la OMS, la Organización de las Naciones Unidas para la Agricultura y la Alimentación y el Fondo de las Naciones Unidas para la Infancia lo utilizan en sus informes con fines de comparación internacional. Se ha propuesto la proporción de niños menores de cinco años cuyo peso para la edad es menor a -2 desviaciones estándar respecto a la población de referencia, como el patrón de comparación más adecuado.

La deficiencia de la talla para la edad se correlaciona consistentemente con el efecto acumulativo de la desnutrición crónica, pero no permite discriminar adecuadamente entre desnutridos actuales y desnutridos anteriores que ya se hayan adaptado o recuperado.

El indicador peso para la talla se correlaciona con la desnutrición aguda, pero no es aconsejable su uso en poblaciones que hayan sido extensamente afectadas en su crecimiento por el efecto de la desnutrición crónica, ya que la proporción de los segmentos corporales superior e inferior se encontrará distorsionada, lo cual inutiliza los parámetros de la población de referencia, basada, obviamente, en niños con crecimiento y relación de segmentos normales..$^{19}$ Como era de esperarse, la distribución geográfica de la prevalencia de desnutrición de acuerdo con este indicador es incongruente con lo que realmente ocurre en el 


\section{Cuadro v \\ Distribución del estado de nUtrición en la población de 1-5 años de acuerdo con el estimador peso para la edad. Comparación de las encuestas nacionales de alimentación 1974, 1979, 1989 y 1996}

\begin{tabular}{|c|c|c|c|c|c|c|c|c|c|c|c|c|c|c|c|c|}
\hline \multirow{3}{*}{ Zona 89} & \multicolumn{16}{|c|}{ Desnutrición } \\
\hline & \multicolumn{4}{|c|}{ Normal } & \multicolumn{4}{|c|}{ Leve } & \multicolumn{4}{|c|}{ M oderada y severa } & \multicolumn{4}{|c|}{$\mathrm{N}$} \\
\hline & $\overline{1974}$ & 1979 & 1989 & 1996 & $\overline{1974}$ & 1979 & 1989 & 1996 & $\overline{1974}$ & 1979 & 1989 & 1996 & $\overline{1974}$ & 1979 & 1989 & 1996 \\
\hline 1 Frontera & 66.90 & 70.00 & 78.20 & 90.91 & 27.50 & 23.00 & 15.90 & 7.95 & 5.50 & 6.90 & 5.90 & 1.14 & 552 & 404 & 239 & 88 \\
\hline 2 Baja california & 84.20 & 77.50 & 76.60 & 78.54 & 13.20 & 15.50 & 13.60 & 11.71 & 2.60 & 7.00 & 6.80 & 9.76 & 257 & 342 & 176 & 205 \\
\hline 3 N oroeste & 68.30 & 76.00 & 84.00 & 79.23 & 26.20 & 18.00 & 12.60 & 14.21 & 5.50 & 6.00 & 3.50 & 6.56 & 517 & 467 & 318 & 366 \\
\hline 4 Norte & 64.70 & 73.00 & 53.20 & 69.68 & 29.40 & 19.90 & 31.70 & 22.78 & 6.00 & 7.10 & 15.20 & 7.54 & 539 & 478 & 158 & 597 \\
\hline $5 \mathrm{~N}$ orte centro & 68.20 & 64.20 & 68.50 & 67.08 & 25.50 & 22.40 & 24.20 & 23.28 & 6.30 & 13.40 & 7.30 & 9.65 & 550 & 411 & 302 & 653 \\
\hline 6 N oreste & 62.20 & 67.70 & 67.40 & 68.88 & 29.90 & 24.70 & 27.50 & 23.43 & 7.90 & 7.60 & 5.10 & 7.69 & 304 & 198 & 138 & 286 \\
\hline 7 Pacífico norte & 66.00 & 47.30 & 69.20 & 69.85 & 23.90 & 29.30 & 25.60 & 21.23 & 10.20 & 23.30 & 5.20 & 8.91 & 395 & 558 & 458 & 617 \\
\hline 8 Centro norte & 58.20 & 40.40 & 52.40 & 64.58 & 31.00 & 35.60 & 36.00 & 22.53 & 10.80 & 24.00 & 11.60 & 12.89 & 232 & 292 & 267 & 830 \\
\hline 90 ccidente & 65.20 & 67.90 & 66.30 & 69.85 & 25.80 & 24.30 & 25.90 & 20.31 & 9.00 & 7.80 & 7.80 & 9.83 & 539 & 717 & 965 & 1088 \\
\hline 10 Bajío & 41.40 & 48.50 & 59.00 & 57.73 & 38.30 & 31.10 & 28.40 & 26.37 & 20.40 & 20.20 & 12.70 & 15.90 & 596 & 1198 & 1460 & 2799 \\
\hline 11 Huasteca y sierra & 42.40 & 44.70 & 31.10 & 44.83 & 37.50 & 27.30 & 35.20 & 29.97 & 20.10 & 28.00 & 33.70 & 25.20 & 184 & 940 & 1263 & 2619 \\
\hline 12 Altiplano norte & 55.80 & 42.30 & 53.90 & 55.91 & 29.30 & 31.90 & 30.70 & 30.03 & 14.90 & 25.80 & 15.40 & 14.06 & 385 & 803 & 1051 & 1735 \\
\hline 13 Sureste & 43.90 & 42.40 & 53.10 & 42.89 & 37.40 & 34.60 & 30.40 & 31.33 & 18.70 & 23.00 & 16.50 & 25.78 & 707 & 870 & 1061 & 2560 \\
\hline 14 Altiplano este & 37.40 & 40.70 & 60.10 & 44.32 & 44.30 & 38.60 & 23.90 & 30.12 & 18.60 & 20.70 & 16.00 & 25.56 & 249 & 484 & 597 & 1162 \\
\hline 15 Golfo centro & 44.80 & 38.40 & 45.50 & 49.87 & 34.30 & 31.10 & 30.70 & 31.16 & 20.90 & 30.40 & 23.70 & 18.97 & 229 & 739 & 628 & 1149 \\
\hline 16 Mixteca y cañada & 18.50 & 32.30 & 29.60 & 38.01 & 40.20 & 27.10 & 30.50 & 32.29 & 41.20 & 40.70 & 39.90 & 29.70 & 264 & 1060 & 1369 & 1468 \\
\hline 17 Pacífico sur & 30.30 & 33.20 & 30.70 & 44.93 & 38.70 & 25.40 & 36.00 & 32.53 & 31.00 & 41.40 & 33.30 & 22.54 & 133 & 401 & 573 & 1242 \\
\hline 18 Golfo sureste & 47.00 & 47.50 & 45.80 & 51.07 & 38.80 & 30.50 & 34.50 & 31.72 & 14.20 & 22.00 & 19.70 & 17.21 & 249 & 550 & 640 & 2522 \\
\hline 19 Península de Yucatán & 50.70 & 47.30 & 32.80 & 38.97 & 32.20 & 32.40 & 37.40 & 33.38 & 17.20 & 20.30 & 29.80 & 27.65 & 227 & 586 & 728 & 1432 \\
\hline $\mathrm{N}$ acional & 49.30 & 49.80 & 52.70 & 52.17 & 33.30 & 28.40 & 28.30 & 28.55 & 17.40 & 21.90 & 19.00 & 19.28 & 7108 & 11498 & 12391 & 23418 \\
\hline
\end{tabular}

Fuente: Instituto N acional de la N utrición Salvador Zubirán, EN AL

país; el peso para la talla no resulta muy adecuado para evaluar el estado de nutrición de la población rural mexicana, sobre todo en los estados con alta prevalencia de desnutrición.

Los puntos de corte en porcentajes propuestos en la clasificación de Gómez del peso para la edad son estadísticamente menos consistentes que el uso de la puntuación $Z$; sin embargo, por su extensa utilización en años anteriores, es conveniente referir la prevalencia de desnutrición con esta clasificación para posibles comparaciones con encuestas que en el pasado hayan informado de esta manera sus resultados.

La prevalencia de desnutrición de la población prescolar que notificó la ENAL-96 pone de manifiesto que ésta sigue siendo un grave problema de salud pública en el medio rural mexicano. Bajo la hipótesis de que la prevalencia de desnutrición es similar en las localidades rurales de menos de 500 habitantes, la desnutrición de alto riesgo (grados moderado y severo) estaría afectando a cerca de 700000 niños menores de cinco años del medio rural.
Respecto a la distribución geográfica, se hace evidente la polarización norte-sur de la desnutrición en México. En general los estados del norte y el de Jalisco muestran niveles de desnutrición mínimos; la mayoría de los estados del centro de la República y el de Tabasco presentan niveles moderados, mientras que en los estados del sureste la desnutrición alcanza niveles superiores. Esta distribución parece estar asociada a la extensa presencia de grupos indígenas en los estados con mayor prevalencia de desnutrición. Subsiste la polémica acerca de la pertinencia de los indicadores peso para la edad y talla para la edad, así como de la población de referencia, utilizados para evaluar el estado de nutrición de los niños indígenas. Se ha discutido mucho en qué medida la baja talla observada en poblaciones indígenas es producto de la mala nutrición, o bien, del somatogenotipo. A pesar de que no hay estudios concluyentes, existen evidencias de que la población indígena mejor alimentada tiende a crecer en forma similar a la norma de referencia. ${ }^{20}$ Para fines de planeación en salud y nutrición públicas, esta polémica 
puede obviarse; la ENAL-96 muestra claramente cuáles son los estados con mayores problemas de desnutrición, en los que es necesario dar prioridad a la atención de los grupos identificados en mayor riesgo y daño. Cabe señalar que, epidemiológicamente, la magnitud de la prevalencia de desnutrición observada en las comunidades indígenas constituye una situación de alarma que demanda una atención inmediata.

Sin duda la explicación última acerca de la magnitud y distribución de la desnutrición en México se encuentra en el grave rezago social que afecta al medio rural. En el cuadro VI se muestra la prevalencia de algunos de los indicadores de calidad de vida, recabados por la ENAL-96, en los cinco estados con mayor prevalencia de desnutrición respecto a todo el país, y se destaca el estado con valores más altos para dicho indicador. El estado de Guerrero ocupa los últimos lugares en la mayoría de los indicadores: $70 \%$ de las familias del medio rural guerrerense habitan en viviendas de mala calidad; $58 \%$ carecen de agua potable; $55 \%$ de las madres de familia son analfabetas; el gasto per cápita en alimentos es de tan sólo cuatro pesos diarios; dos de cada cinco familias jamás consumen leche, dos de cada tres niños están desnutridos, y uno de cada tres padece desnutrición de alto riesgo. Yucatán, Puebla, Oaxaca y Chiapas también mostraron ni- veles de gran deterioro en estos indicadores, mientras que los estados con mejores condiciones de vida son los que presentan menor incidencia de desnutrición infantil. Resulta sorprendente que a lo largo de 22 años la prevalencia de desnutrición de los prescolares registrada en la serie ENAL casi no presente variación en un plano global. La mejoría en algunas regiones se ha acompañado del deterioro en otras; el mayor deterioro se ha observado generalmente en los estados en peores condiciones. La persistencia de una elevada prevalencia de desnutrición en el medio rural mexicano obliga a reflexionar acerca de la eficacia de los programas de asistencia social alimentaria y combate a la desnutrición realizados en las dos décadas recientes, y hace evidente la urgente necesidad de su reorientación.

\section{Referencias}

1. Fondo de las $\mathrm{N}$ aciones U nidas para la Infancia. El Progreso de las $\mathrm{N}$ aciones 1996. Nueva York: UN ICEF House, 1996:23.

2. Colombo M, López I, DeAndraca I. Desnutrición grave precoz y desarrollo psicomotor. Efectos de un programa de rehabilitación. Arch Latinoam N utr 1993:43(2):146-150.

3. Rivera J, Long K, G onzález-C osio T, Parra S, Rivera M, Rosado JL. N utrición y salud. México, D.F. : Cuadernos de Salud, Secretaría de Salud, 1994.

\section{Cuadro VI \\ Condiciones de VIVIENDA, EDUCACIÓN, NUTRICIÓN Y ALIMENTACIÓn EN LOS CINCo ESTAdos CON MAYOR PREVALENCIA DE DESNUTRICIÓN Y EL ESTADO EN MEJORES CONDICIONES}

\begin{tabular}{|c|c|c|c|c|c|c|c|}
\hline Condición & Guerrero & Yucatán & Puebla & Oaxaca & Chiapas & Nacional & Estado en mejor condición \\
\hline Viviendas con piso de tierra (\%) & $57.5^{*}$ & 27.6 & 51.2 & 56.0 & 56.2 & 36.7 & Chihuahua (8.0) \\
\hline Viviendas sin agua potable (\%) & $57.6 *$ & 30.0 & 46.7 & 51.5 & 42.8 & 44.1 & Aguascalientes (2.6) \\
\hline Viviendas de calidad mala y muy mala (\%) & $69.0 *$ & 36.8 & 57.6 & 62.6 & 59.3 & 41.9 & Aguascalientes (6.7) \\
\hline Padre analfabeto $(\%)$ & $53.4^{*}$ & 46.7 & 43.1 & 41.6 & 46.7 & 38.5 & Chihuahua (13.3) \\
\hline Madre analfabeta (\%) & $54.7^{*}$ & 53.1 & 44.9 & 45.7 & 51.6 & 37.9 & Chihuahua (11.7) \\
\hline \multicolumn{8}{|l|}{$\mathrm{N}$ iños desnutridos } \\
\hline Peso/edad (\%) & $63.1^{*}$ & 61.6 & 53.3 & 54.5 & 49.6 & 42.8 & Sonora (13.0) \\
\hline \multicolumn{8}{|l|}{$\mathrm{N}$ iños desnutridos } \\
\hline Talla/edad (\%) & $71.3^{*}$ & 73.1 & 66.5 & 69.9 & 71.6 & 55.9 & Baja C alifornia (22.3) \\
\hline $\mathrm{N}$ iños desnutridos de alto riesgo (\%) & $32.4^{*}$ & 28.7 & 25.4 & 23.3 & 20.00 & 16.9 & Sonora (4.4) \\
\hline Gasto en alimentos (pesos per cápita/semana) & 28.6 & 29.3 & 25.2 & 27.9 & $21.9 *$ & 30.1 & Baja California S. (70.1) \\
\hline Consumo per cápita de leche (ml) & 72.7 & $44.1^{*}$ & 77.2 & 101.0 & 60.0 & 125.0 & Jalisco (255.7) \\
\hline Familias que no consumen leche (\%) & 38.00 & 54.7 & $55.1^{*}$ & 46.4 & 51.0 & 35.0 & Chihuahua (8.0) \\
\hline \multicolumn{8}{|c|}{$\begin{array}{l}\text { * Estado en peor condición respecto a to do el país } \\
\text { Fuente: Instituto N acional de la N utrición Salvador Zubirán, EN AL-96 }\end{array}$} \\
\hline
\end{tabular}


4. Comisión Interinstitucional. Programa de Alimentación y N utrición Familiar. México: Gobierno de la República, 1995.

5. Chávez A, Avila A, Roldán JA, Bermejo S, Madrigal $H$. The food and nutrición situation in Mexico, a report of the 1960-1990 tendencies. México, D.F.: Instituto N acional de la N utrición Salvador Zubirán, 1992:1-10. 6. Chávez $A$, ed. Encuestas $N$ utricionales en México. 2a. edición. División de N utrición de Comunidad. Publicación L-1. México, D.F.:Instituto N acional de la N utrición Salvador Zubirán, 1974:2.

7. Bassols A. Geografía económica de México. México, DF:Trillas, 1972: 400-418.

8. Madrigal H, Moreno O, Chávez A. Encuesta N acional de Alimentación 1979. Resultados de la encuesta rural analizada por entidad federativa y desagregada según zonas nutricionales. México, D.F.: División de Nutrición, Instituto N acional de la Nutrición, 1982.

9. Madrigal H, Chávez A, Moreno O, García T, Gutiérrez G. Consumo de alimentos y estado de nutrición de la población del medio rural mexicano. Rev Invest Clin 1986;38supl:9-20.

10. Secretaría de Salud. Dirección General de Epidemiología. Encuesta $\mathrm{N}$ acional de $\mathrm{N}$ utrición 1988. Resultados $\mathrm{N}$ acionales y por Regiones. México, D.F.: DGE-SSA, 1988.

11. Instituto N acional de la N utrición Salvador Zubirán. Encuesta N acional de Alimentación en el Medio Rural EN AL-89. México, D.F.: IN N SZ, Comisión Nacional de Alimentación, 1990.
12. Avila-C uriel A, C hávez-Villasana A, Shamah-Levy T, Madrigal-Fritsch $H$. La desnutrición infantil en el medio rural mexicano: análisis de las encuestas nacionales de alimentación. Salud Publica Mex 1993;35:658-666.

13. Sistema Nacional para el Desarrollo Integral de la Familia. Primer Censo N acional de Talla. México, D.F.: DIF/SEP, 1994.

14. Sistema Nacional para el Desarrollo Integral de la Familia. II Censo N acional de Talla, 1994. México, D.F.: DIF/SEP, 1997.

15. A vila A, Shamah T, Chávez A. Encuesta U rbana de Alimentación y N utrición en la Zona Metropolitana de la Ciudad de México. México, D.F.: IN N SZ, 1995.

16. Habitch JP. Estandarización de métodos epidemiológicos cuantitativos sobre el terreno. Bol 0 ficina Sanit Panam 1974;76:375-384.

17. World Health 0 rganization. Measuring change in nutritional status. Ginebra: W HO, 1983.

18. Statistical Program for Social Sciences. SPSS.Ver 6.1. Chicago, Illinois: 24 junio, 1994.

19. Dibley MJ,Goldsby JB, Staehling N W, Trowbritge FL. D evelopment of normalized courves for the international growth reference: H istorical and technical considerations. Am J N utr 1987;46:736-748.

20. Avila-Curiel A. Nutrición, crisis y migración. Memorias del Cuarto Simposio de N utrición y Alimentos. México, D.F.: U niversidad Iberoamericana, 1991:174-187. 